\title{
Re: Surgery Versus Radiotherapy for Clinically-localized Prostate Cancer: A Systematic Review and Meta-analysis
}

\author{
Wallis CJ1, Saskin R2, Choo R3, Herschorn S4, Kodama RT4, Satkunasivam R4, Shah PS5, Danjoux \\ C6, Nam RK7
}

1 Division of Urology, Sunnybrook Health Sciences Centre, Sunnybrook Research Institute, Toronto, Canada; Division of Urology, Department of

Surgery, University of Toronto, Toronto, ON, Canada; Institute of Health Policy, Management and Evaluation, University of Toronto, Toronto, ON, Canada.

2University of Toronto Faculty of Medicine, Institute of Health Policy, Division Management and Evaluation, Toronto, Canada; Sunnybrook Research Institute, Sunnybrook Health Sciences Centre, Clinic of Urology, Toronto, Canada

3 Mayo Clinic, Clinic of Radiation Oncology, Rochester, USA

4Sunnybrook Research Institute, Sunnybrook Health Sciences Centre, Clinic of Urology, Toronto, Canada; University of Toronto Faculty of Medicine, Department of Surgery, Clinic of Urology, Toronto, Canada

5 University of Toronto Faculty of Medicine, Institute of Health Policy, Division Management and Evaluation, Toronto, Canada; Mount Sinai

Hospital, Clinic of Pediatrics, Toronto, Canada; University of Toronto Faculty of Medicine, Department of Pediatrics, Toronto, Canada

6University of Toronto Faculty of Medicine, Department of Radiation Oncology, Toronto, Canada

7Sunnybrook Research Institute, Sunnybrook Health Sciences Centre, Clinic of Urology, Toronto, Canada; University of Toronto Faculty of

Medicine, Department of Surgery, Clinic of Urology, Toronto, Canada; University of Toronto Faculty of Medicine, Institute of Health Policy,

Division Management and Evaluation, Toronto, Canada

Eur Urol 2016;70:21-30. doi: 10.1016/j.eururo.2015.11.010.

\section{EDITORIAL COMMENT}

Radical treatment options for patients with clinically-localized prostate cancer include radical prostatectomy and radiotherapy. Since there is no level 1 evidence comparing the efficacy of these two modalities, Wallis and coworkers conducted a systematic review and meta-analysis to compare the data on overall and prostate cancer-specific survival among patients treated with radiotherapy or radical prostatectomy for clinically-localized prostate cancer. Nineteen studies were selected and up to 118.830 patients were pooled. Of note, only two studies provided "dose-escalated" external beam radiotherapy treatments to all radiotherapy patients. The risk of overall (10 studies, aHR $1.63,95 \%$ confidence interval $1.54-1.73, p<0.00001 ;\left.\right|^{2}=0 \%$ ) and prostate cancer-specific (15 studies, aHR 2.08 , $95 \%$ confidence interval $1.76-2.47, p<0.00001 ;\left.\right|^{2}=48 \%$ ) mortality were higher for patients treated with radiotherapy compared with those treated with surgery. Subgroup analyses by risk group, radiation regimen, time period, and follow-up length did not alter the direction of results. They concluded that radiotherapy for prostate cancer is associated with an increased risk of overall and prostate cancer-specific mortality compared with surgery based on observational data. The methodological limitations of observational studies should be considered while interpreting the results. The ProtecT trial and SPCG-15 trial comparing radical prostatectomy to radiotherapy among patients with low/intermediate and high-risk cancer respectively are awaited.

Özgür Yaycıoğlu, MD 